\title{
Lung injury associated with inhalation of effective microorganism blends
}

\author{
Jee-min Kim', Yoon Jin Kwak², Ho II Yoon ${ }^{3,4}$ \\ ${ }^{1}$ Division of Pulmonary and Critical Care Medicine, Department of Internal Medicine, National Medical Center, Seoul; Departments of ${ }^{2}$ Pathology and \\ ${ }^{3}$ Internal Medicine, Seoul National University College of Medicine, Seoul; ${ }^{4}$ Department of Pulmonary and Critical Care Medicine, Seoul National University \\ Bundang Hospital, Seongnam, Korea
}

Since 2009, effective microorganisms (EMs) have been supplied by the local government to the citizens of Seongnam, Korea, for various environment-protective uses including manufacturing detergents, cosmetics and humidifier disinfectants. A 68-year-old man who had placed an EM blends into a humidifier for inhalation visited the emergency room with complaints of fever and dyspnea. He was in a shock state with hypoxia. Chest computed tomography revealed diffuse ground-glass opacities that were dominant in the bilateral upper lobes. Fiberoptic bronchoscopy with bronchoalveolar lavage and transbronchial lung biopsy was performed. Bronchoalveolar lavage fluid analysis and biopsy findings were consistent with alveolar hemorrhage. All microbiological and virological test results were negative. His symptoms and radiographic opacities had improved markedly after several days of conservative care, and he was discharged healthy after 1 week of hospital stay.

Key Words: acute lung injury; haemorrhage; inhalation; microorganism fermentation extract

Effective microorganisms (EMs) are various blends of predominantly anaerobic microorganisms in a carbohydrate-rich liquid carrier substrate [1]. Although composition of EM varies depending on its components, the major microbial species are lactic acid, photosynthetic bacteria and various yeasts [2]. It was believed that the discharge of EM blends into rivers would help clean the environment by eliminating green algae and reduce foul odors by accelerating aerobic breakdown $[1,3]$.

As part of the social agenda to purify sewage in Korea, EM blends have been supplied by the local government to the citizens of Seongnam since 2009. Their use has not only been limited to cleaning water but has also broadened into manufacturing soap, shampoo, dish cleaner, and humidifier detergents. We report the case of a man who presented with impending respiratory failure after inhaling vaporized EM blends from a humidifier.

\section{CASE REPORT}

A previously healthy 68-year-old man visited the emergency room (ER) for aggravated symptoms of cough and dyspnea for 3 days. He initially visited a local clinic and was prescribed antitussives and empirical oral antibiotics; however, his symptoms aggravated, with spiking

\section{Case Report}

Received: October 19, 2018 Revised: March 14, 2019

Accepted: March 31, 2019

\section{Corresponding author}

Ho II Yoon

Division of Pulmonary and Critical Care Medicine, Department of Internal Medicine, Seoul National University Bundang Hospital, Seoul National University College of Medicine, 82 Gumi-ro 173beongil, Bundang-gu, Seongnam 13620, Korea

Tel: +82-31-787-7036

Fax: +82-31-787-4052

E-mail:dextro70@gmail.com

Copyright (๑) 2020 The Korean Society of Critical Care Medicine

This is an Open Access article distributed under the terms of Creative Attributions Non-Commercial License (https:// creativecommons.org/li-censes/by-nc/4.0/) which permits unrestricted noncommercial use, distribution, and reproduction in any medium, provided the original work is properly cited. 
fever up to $38.6^{\circ} \mathrm{C}$. At the ER visit, he had an acutely ill-looking appearance and claimed general weakness with dyspnea. His initial blood pressure was $97 / 50 \mathrm{mmHg}$, and heart rate was 106 beats/min. His respiration was shallow and fast, and he had hypoxia, with an oxygen saturation of $91 \%$ on pulse oximetry on room air. Hypoxemia was corrected after oxygen supplementation with nasal prong $(6 \mathrm{~L} / \mathrm{min})$, while hypotension was corrected with administration of norepinephrine (0.15 $\mu \mathrm{g} / \mathrm{kg} / \mathrm{min}$ ) with fluid resuscitation. Coarse breathing sounds with inspiratory crackles in whole lung field were noted. $\mathrm{Al}$ though C-reactive protein was mildly elevated $(3.47 \mathrm{mg} / \mathrm{dl})$, there was no leukocytosis or elevated neutrophil counts (white blood cell, 5,900/ $\mu \mathrm{l}$ [segment $60 \%$ ]). There were no other serological laboratory abnormalities including coagulation, cardiac markers, and brain natriuretic peptide levels. Chest radiography revealed a large consolidation in the right middle lung field, and chest computed tomography revealed ill-defined ground-glass opacities with consolidations in the right upper,
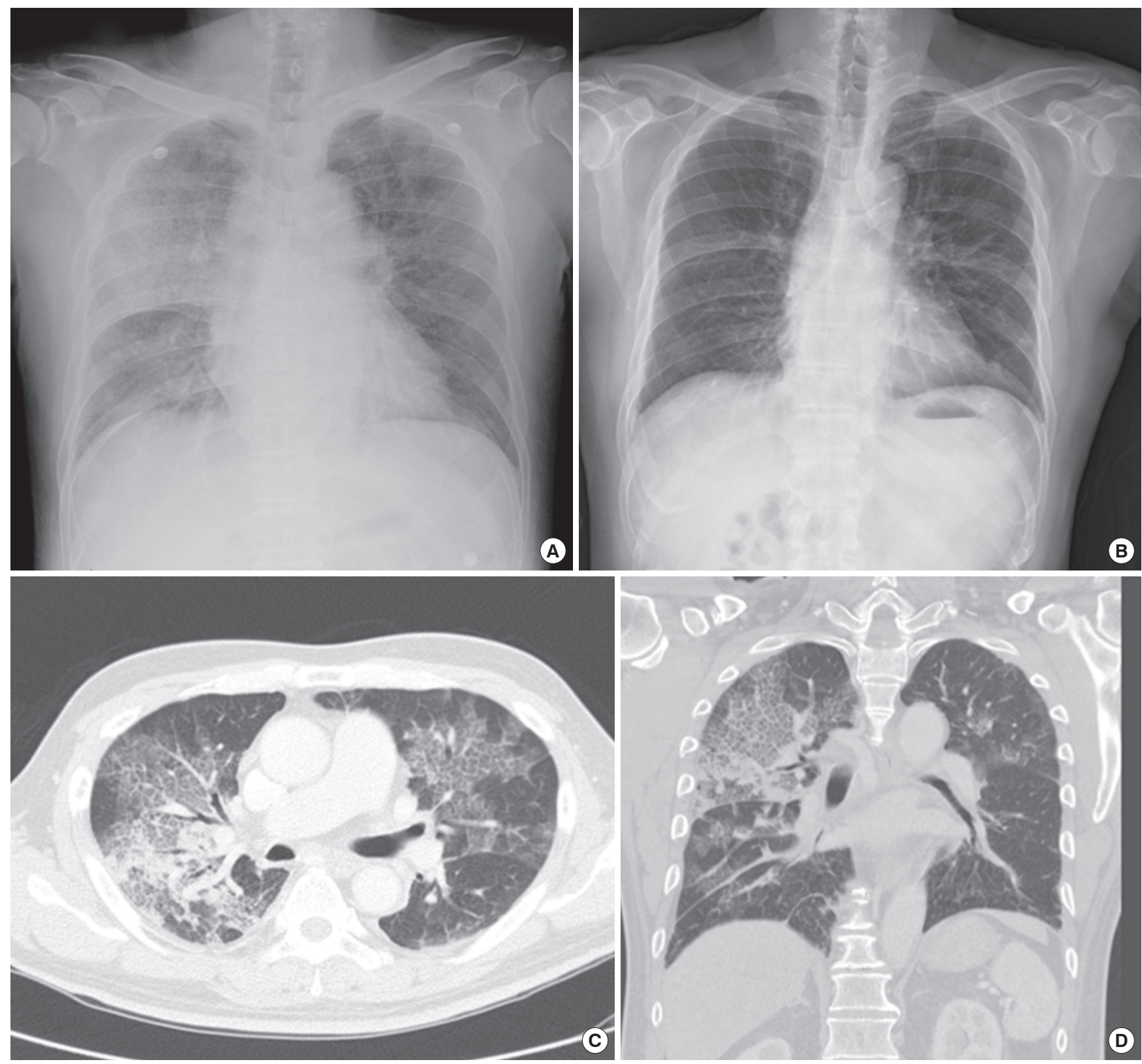

Figure 1. (A) Initial chest X-ray showing dense consolidation in right upper and middle lung field. (B) Chest X-ray on hospital day 6 revealing the resolution of consolidative lesions. (C, D) Chest computed tomography showing upper lobe-dominant consolidation with bilateral ground-glass opacities. 

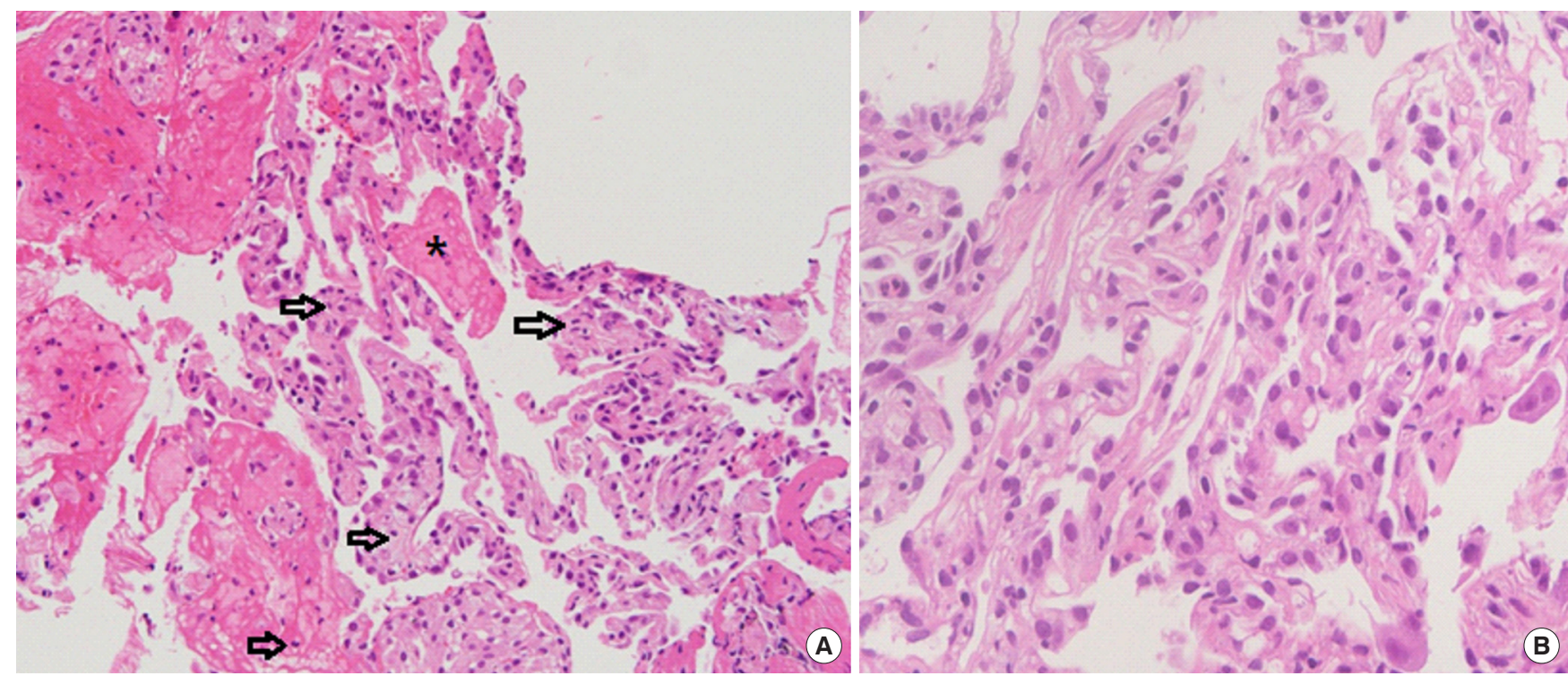

Figure 2. Transbronchial lung biopsy of right upper lobe. (A) Many fibrin deposits (asterisk) within alveolar space which is consistent with alveolar hemorrhage $\left(H \& E_{1} \times 200\right)$. Deposition of inflammatory cells (arrows) in interstitium is also noted. (B) Alveoli with capillaries showing that there is no evidence of vasculitis (right; $\mathrm{H} \& \mathrm{E}_{1} \times 400$ ).

right middle, and left upper lobes (Figure 1).

The patient was an office worker with no recent history of travel. He had no relevant history of taking medications or health foods. He had used EM fermented blends inside a humidifier and inhaled it overnight a day before he developed symptoms. Instructions limit the use of EM blends to humidifier disinfectants, but the patient had added the blend into a humidifier for inhalation, believing it would be beneficial to his health.

Initial treatment, including intensive intravenous fluid administration, empirical antibiotics and antiviral therapy was administered for septic shock due to possible community-acquired bacterial or viral pneumonia. There was a high prevalence of influenza at the time he presented to the ER.

Flexible bronchoscopy was performed with bronchoalveolar lavage and transbronchial lung biopsy from the right upper lobe. The retrieved fluid was grossly bloody and sequential aliquots exhibited more hemorrhage, which was consistent with alveolar hemorrhage. The biopsy specimen finding was also suggestive of alveolar hemorrhage (Figure 2). Cytospin results from the retrieved fluid were as follows: red blood cell, 29,750/ $\mu \mathrm{l}$; white blood cell, $550 / \mu \mathrm{l}$ (macrophage 33\%, segmented neutrophils $44 \%$, lymphocytes $14 \%$, eosinophils $4 \%$ ). All microbiological study results were negative, including bacteria and fungus, virus and tuberculosis.

Considering the temporal relationship between the inhalation of EM blends and the presentation of acute lung injury, and the spatial relationship in which the lesion exhibited upper lobe dominancy, negative microbiological results, and bronchoscopic and pathological findings were consistent with diffuse alveolar hemorrhage. It was concluded that the patient sustained an acute inhalation lung injury caused by the EM vapor. Initially, empirical antibiotics were administered for few days; however, after the clinical diagnosis of EM-related lung injury, these were discontinued. His symptoms and radiographic opacities improved markedly after several days of conservative care. On hospital day 6, chest radiography revealed the complete resolution of consolidative lesions and he was free of respiratory symptoms (Figure 1). He was discharged healthy and is currently visiting an outpatient clinic regularly with no residual symptoms or sequelae.

\section{DISCUSSION}

The concept of EM was first proposed in the 1980s. It was typically manufactured for environmental applications, especially agricultural use [1]. Consisting of various species of microbes that exist naturally in the environment, its use was initially aimed at cleaning septic tanks in farms [4]. Its use has broadened to clearing polluted environments, including rivers, and has been used by many local governments in Korea for social agenda projects to purify sewage and remove foul odor from rivers. Since 2009, nearly 39 tonnes of EM material has been released into the stream current in Seongnam every week. 
Not only limited to purifying water, the use of EM materials has also been widened to manufacturing detergents, disinfectants, cosmetics, and plant fertilizers.

To our knowledge this is the first report of acute lung injury caused by inhalation of EM vapor. The patient did not discard the EM blends after cleaning the humidifier but continued to vaporize and inhale because he believed it was good for his health. Symptoms of cough and dyspnea developed the day after EM use. He presented with shock and impending type 1 respiratory failure on the day of arrival to our hospital. Absence of leukocytosis, low levels of inflammatory markers, negative microbiological results, and lack of response to empirical antibiotics all suggested acute lung injury associated with inhalation of EM vapor. Bronchoalveolar lavage fluid and biopsy were altogether consistent with alveolar hemorrhage with no evidence of vasculitis.

Approximately a decade ago, there was a cluster of fatal lung injuries associated with the use of humidifier disinfectant in Korea. Three chemicals-oligo ethoxyethyl guanidinium chloride, polyhexamethylene guanidine phosphate, and didecyldimethylammonium chloride-were believed to be the causative agents of lung toxicities; nevertheless, the exact mechanism and processes of lung injury remain unclear [5]. This case mimics interstitial lung disease after the use of humidifier disinfectant, but differs from lung injuries after chemical disinfectant in that it exhibited acute onset of symptoms after a short period of EM blend use, presented diffuse alveolar hemorrhage, and had a relatively quick recovery after conservative care. Radiological findings of humidifier disinfectant injury differ from those of EM blend in that lung injury related to humidifier disinfectants usually exhibits initial multifocal and patchy consolidation in the lower lobes with subpleural sparing, and later diffuse centrilobular ground-glass opacities involving the entire lung. Pathological findings also differ in that humidifier disinfectant-associated lung injuries usually exhibit fibroinflammatory processes, which predominantly involve the bronchioles and centrilobular lung parenchyma, with no notable granulomas [6]. Lung injury after inhalation of EM vapor may be due to toxic chemicals produced in the process of fermentation, microbes in the EM liquor, or other preservatives or additives. A decade ago, Do et al. [7] reported that EM extract attenuates airway hyperreactivity and inflammation through the inhibition of T-helper cell 2 response in mouse models. The patient in this case is confronted with this case, implying that in vivo inflammatory reactions in humans may differ from those in animals; alternatively, there may be a variability in dose responses. Further studies investigating EM vapors and lung toxicities are needed.

In conclusion, the use of EM blends in humidifier may cause acute inhalation injury and be serious enough to cause respiratory failure. Cautions and recommendations for proper use should be provided for its safe use, and further investigations should be performed to clarity the relationships between EM vapor and its toxicities in the lungs.

\section{CONFLICT OF INTEREST}

No potential conflict of interest relevant to this article was reported.

\section{ORCID}

Jee-min Kim https://orcid.org/0000-0003-3357-5436

Yoon Jin Kwak https://orcid.org/0000-0001-5314-2465

Ho Il Yoon https://orcid.org/0000-0002-5306-3692

\section{AUTHOR CONTRIBUTIONS}

Conceptualization: HIY, JMK. Data curation: HIY, YJK. Writing - original draft: HIY, JMK. Writing-review \& editing: HIY, JMK, YJK.

\section{REFERENCES}

1. Higa T, Parr JF. Beneficial and effective microorganisms for a sustainable agriculture and environment. Atami: International Nature Farming Research Center; 1994.

2. Higa T, Wididana GN. The concept and theories of effective microorganisms. Proceedings of the first international conference on Kyusei Nature Farming. Washington, DC: US Department of Agriculture; 1991.

3. Abdel-Shafy HI, AL-Sulaiman AM, Mansour MS. Greywater treatment via hybrid integrated systems for unrestricted reuse in Egypt. J Water Process Eng 2014;1:101-7.

4. Szymanski N, Patterson RA. 2003 Effective microorganisms (EM) and wastewater systems in future directions for on-site systems: best management practice. In: Patterson RA, Jones MJ, editors. Proceedings of On-site '03 Conference; 2003 Sep 30-Oct 2; Armidale, Australia. Armidale: Lanfax Laboratories Armidale; 2003. p. 347-54.

5. Paek D, Koh Y, Park DU, Cheong HK, Do KH, Lim CM, et al. Nationwide study of humidifier disinfectant lung injury in South Korea, 1994-2011: incidence and dose-response relationships. Ann Am Thorac Soc 2015;12:1813-21. 
6. Kim WY, Hong SB. Humidifier disinfectant-associated lung injury: six years after the tragic event. Tuberc Respir Dis (Seoul) 2017;80:351-7.

7. Do JS, Seo HJ, Hwang JK, Kim JH, Nam SY. Effective microor- ganism fermentation extract (EM-X) attenuates airway hyperreactivity and inflammation through selective inhibition of the TH2 response independently of antioxidant activity. Int J Mol Med 2007;20:631-5. 